\title{
Precise radial velocities with BOES ${ }^{\star}$ Detection of low-amplitude pulsations in the K-giant $\alpha$ Arietis
}

\author{
K. M. Kim ${ }^{1}$, D. E. Mkrtichian ${ }^{2,3}$, B.-C. Lee ${ }^{1,4}$, Inwoo Han ${ }^{1}$, and A. P. Hatzes ${ }^{5}$ \\ 1 Korea Astronomy and Space Science Institute, 61-1 Whaam-Dong Yuseong-Gu, Daejeon 305-348, Korea \\ 2 Astrophysical Research Center for the Structure and Evolution of the Cosmos, Sejong University, Seoul 143-747, Korea \\ e-mail: david@arcsec.sejong.ac.kr \\ 3 Astronomical Observatory, Odessa National University, Shevchenko Park, Odessa 65014, Ukraine \\ 4 Department of Astronomy and Atmospheric Sciences, Kyungpook National University, Daegu 702-701, Korea \\ 5 Thüringer Landessternwarte Tautenburg, 07778 Tautenburg, Germany
}

Received 16 March 2005 / Accepted 26 January 2006

ABSTRACT

\begin{abstract}
We present the first results from a high-precision radial velocity study of the K2 III giant star $\alpha$ Ari. Observations were acquired over 6 nights in 2004 using the new high-resolution spectrograph BOES (Bohyunsan Observatory Echelle Spectrograph) of the $1.8-\mathrm{m}$ telescope. A high radial-velocity precision was achieved by using the high-resolution $(R=90000)$ mode of BOES and an iodine gas absorption cell. The radial velocity measurements made during JD $=2452948-2452950$ show coherent, low-amplitude variations with a period of $P_{1}=0.571$ days (or aliases at 0.445 or 0.821 days), and an amplitude of $18.9 \mathrm{~m} \mathrm{~s}^{-1}$. Observations of $\tau$ Cet over this same interval are constant to within $3 \mathrm{~m} \mathrm{~s}^{-1}$. After subtracting the contribution of the 0.57 -day period, we find evidence for a second period, $P_{2}=0.190$ days. Observations made on a second run during JD $=2452975-2452981$ show that the radial velocity variations are indeed present, but on shorter time-scales and with a lower amplitude. Two probable periods fit the radial velocity measurements from the second run reasonably well: 0.185 days or an alias of 0.256 days. The shorter period coincides with the secondary one found in the earlier measurements. We conclude that, similar to other K-giant pulsating stars, $\alpha$ Ari shows unstable acoustic pulsations or mode switching on time scales of tens of days. The calculated pulsation constants for the dominant 0.571-day period is consistent with third-overtone pulsations, while the secondary periodicity found in both data runs corresponds to a high overtone $(n \geq 12)$.
\end{abstract}

Key words. stars: late-type - stars: oscillations

\section{Introduction}

Short-term, periodic radial velocity $(R V)$ oscillations in the K-giant Arcturus were discovered almost 20 years ago (Smith et al. 1987). Walker et al. (1989) subsequently found $R V$ variations in $5 \mathrm{~K}$-giants and one $\mathrm{K}$ supergiant star and proposed that these were a new class of $R V$ variable stars. Additional investigations showed that K-giant stars are multi-periodic on time scales of several hundred days (Hatzes \& Cochran 1993) and several days (Hatzes \& Cochran 1994a). The long-term variations are most likely due to companions or rotational modulation by stellar surface structure.

It is not known if the pulsations in $\mathrm{K}$-giants are coherent on long time scales and there is some evidence that $\mathrm{K}$-giants mode switch (Hatzes \& Cochran 1994a,b). The best-studied K-giant star, Arcturus, showed up to $10 R V$ pulsation modes with periods less than 10 days and equally-spaced in frequency (Merline 1995; Hatzes \& Cochran 1998; Retter et al. 2003). A similar number of modes were found in the K-giant $\alpha$ UMa by Buzasi et al. (2000) using photometric measurements made with the guide camera of the WIRE satellite. The observations of the K-giant GSC 09137-03505 with the Fine Guidance Sensors of the Hubble Space Telescope showed brightness variations corresponding to three approximately equidistant frequencies in

^ Based on observations collected at Bohyunsan Optical Astronomy Observatory. turn corresponding to periods in the range $0.162-0.542$ days (Kallinger et al. 2005). All of these results lend evidence of pulsations in $\mathrm{K}$ giant stars with consecutive acoustic overtones. Thus, K-giant stars are amenable to asteroseismic studies that can directly determine fundamental stellar parameters.

The K2 III star $\alpha$ Ari (HD 12929) is a red giant branch star. This star was one of the K-giant stars for which Walker et al. (1989) reported $R V$ variability, although it showed the lowest amplitude variability and no period could be determined. It is often used as a standard star for calibration of the fundamental parameters of late-type giant stars (e.g. Alonso et al. 1999).

\section{Observations, instrument, and reduction}

The star $\alpha$ Ari was observed within the framework of an international program to use high-precision radial velocity measurements to search for planets and pulsations in K-giants. This program is being carried out at the Bohyunsan Optical Astronomy Observatory (BOAO), Korea, in collaboration with the Astrophysical Research Center for the Structure and Evolution of the Cosmos (ARCSEC, Korea), the Thüringer Landessternwarte Tautenburg (TLS, Germany), and the Astronomical Observatory of Odessa National University (Ukraine)

High-resolution spectra of $\alpha$ Ari and the radial velocity standard $\tau$ Ceti were acquired using the fiber-fed BOES 
(Bohyunsan Observatory Echelle Spectrograph) (Kim et al. 2002), which is a high throughput, versatile, fiber-fed, prism cross-dispersed spectrograph for the 1.8-m telescope at BOAO. Using a $2 \mathrm{k} \times 4 \mathrm{k}$ CCD, BOES has a wavelength coverage of 3600-10500 $\AA$ (86 spectral orders) in one exposure. Three fibers of 80,200 , and $300 \mu \mathrm{m}$ provide projected widths on the sky of $1.1,2.9$, and 4.3 arcsec, respectively. The measured spectrograph resolving power, $R=\lambda / \delta \lambda$, for each respective fiber is $R=90000,45000$ and 30000 . The efficiency can be as high as $15 \%$.

For precise stellar radial velocity $(R V)$ measurements, the instrument was configured to achieve a resolving power $R=$ 90000 (80 $\mu \mathrm{m}$ fiber). Relative $R V$ measurements were made using a velocity metric provided by an iodine $\left(\mathrm{I}_{2}\right)$ absorption cell. Molecular iodine has useful absorption lines in the wavelength interval 5000-6300 . To achieve the highest $R V$ precision, the spatial and temporal variations of the instrumental profile (IP) were modeled using the iodine absorption lines. This was done largely following the procedure outlined in Valenti et al. (1995) and Butler et al. (1996). Each spectral order was divided into 30 spectral chunks and the $R V$ calculated in each chunk. The $R V$ measurements from all spectral chunks were then combined and weighted by the inverse square of the $R V$ standard deviation for each chunk. The rms $R V$ variations of the chunks give us an estimate of the internal error of our $R V$ measurement. This procedure may underestimate the true error since it does not take into account (unknown) systematic errors that may also be present.

\section{Radial velocity measurements}

Table 1 shows the BOES relative $R V$ measurements and $\mathrm{rms}$ scatter of the integrated $R V$ measurements for $\alpha$ Ari. Figure 1 shows the BOES radial velocities of $\alpha$ Ari over the time interval JD $=2452948-$ JD 2452950 (Run 1). Also shown are $R V$ measurements for $\tau$ Cet. This star has been shown by radial velocity surveys to be constant to a level less than $5 \mathrm{~m} \mathrm{~s}^{-1}$ (Endl, private communication). Although the $\tau$ Cet measurements are sparse, they do exclude any large, systematic errors in our $R V$ measurements. The $R V \mathrm{~s}$ for $\alpha$ Ari show a peak-topeak variability of $\approx 40 \mathrm{~m} \mathrm{~s}^{-1}$, while $\tau$ Cet is constant with an rms scatter of $\sigma=3.1 \mathrm{~m} \mathrm{~s}^{-1}$. It is clear that $\alpha$ Ari is a variable star.

Figure 2 shows the $R V$ measurements for $\alpha$ Ari and $\tau$ Ceti over the time interval JD $=2452975-2452981$ (Run 2). During this time the observing conditions were poorer, resulting in fewer observations with lower signal-to-noise ratios. Again, $\tau$ Cet is relatively constant with $\sigma=7.8 \mathrm{~m} \mathrm{~s}^{-1}$. Although it is marginal, $\alpha$ Ari shows some variability. The rms scatter of the observations, $\sigma=13.2 \mathrm{~m} \mathrm{~s}^{-1}$, is comparable to the typical, estimated internal error for an observation. As we shall show below, we believe that $\alpha$ Ari did exhibit radial velocity variations during this time.

\section{Period search}

To search for periodic signals we used the Lomb-Scargle periodogram (Lomb 1976; Scargle 1982) and the standard Discrete Fourier Transform (DFT) for unequally spaced data (Deeming 1975).

The top panel of Fig. 3 shows the Scargle periodogram of the $R V$ measurements for JD $=2452948-\mathrm{JD} 2452950$ time interval (Run 1). Due to the data sampling, the periodogram has strong $1 \mathrm{c} \mathrm{d}^{-1}$ aliases. Possible aliasing frequencies of $1.29,1.76$, and $2.25 \mathrm{c} \mathrm{d}^{-1}$ give comparable fits to the data.

The highest peak in the top panel of Fig. 3 is statistically significant. The false alarm probability (FAP) was assessed using the bootstrap randomization technique (Murdoch et al. 1993; Kürster et al. 1997). The measured $R V$ values were randomly shuffled keeping the observed times fixed. A periodogram was then computed for each "random" data set. The fraction of the random data periodograms with higher power than the true data periodogram is a measure of the probability that random noise could cause a peak of equal power in the periodogram. Using $2 \times 10^{5}$ "shuffles" there was no instance where the random periodogram had higher power than the data periodogram. In fact, the highest Scargle-power ( $S P$, a measure of statistical significance) for the random data was $S P \approx 15$, significantly below the $S P \approx 50$ shown by the data. Therefore the FAP $\ll 10^{-6}$.

Using least-square sine fits, we find the following values for possible periods in the $R V$ data: $f_{1}=1.752 \pm 0.0068 \mathrm{c} \mathrm{d}^{-1}$ $\left(P_{1}=0.571 \pm 0.002\right.$ days $), K_{1}=17.78 \pm 0.58 \mathrm{~m} \mathrm{~s}^{-1} ; f_{1}^{\prime}=2.245 \pm$ $0.068 \mathrm{c} \mathrm{d}^{-1}\left(P_{1}^{\prime}=0.445 \pm 0.014\right.$ days $), K_{1}^{\prime}=18.58 \pm 0.66 \mathrm{~m} \mathrm{~s}^{-1}$; and $f^{\prime \prime}{ }_{1}=1.218 \pm 0.01 \mathrm{~cd}^{-1}\left(P^{\prime \prime}{ }_{1}=0.821\right.$ days $), K^{\prime \prime}{ }_{1}=$ $18.92 \pm 0.85 \mathrm{~m} \mathrm{~s}^{-1}$. Phase-folded radial velocity curves for each period are presented in the panels in Fig. 4 . The $P_{1}=0.571$ day period gives a slightly better fit to the data with an rms scatter about the fit of $\sigma=4.60 \mathrm{~m} \mathrm{~s}^{-1}$. However, the other periods give comparable fits: $\sigma=4.95 \mathrm{~m} \mathrm{~s}^{-1}$ for $P_{1}^{\prime}=0.445$ days and $\sigma=4.85 \mathrm{~m} \mathrm{~s}^{-1}$ for $P^{\prime \prime}{ }_{1}=0.821$ days. Although we consider the $P_{1}=0.571$ day period as the one most likely present, we cannot exclude that a period of 0.445 days or 0.821 days may actually be present due to alias problems.

The Scargle periodograms of residuals after subtracting these $f_{1}=1.752 \mathrm{c} \mathrm{d}^{-1}\left(P_{1}=0.571\right.$ day $)$ or $f_{1}^{\prime}=2.245 \mathrm{c} \mathrm{d}^{-1}$ $\left(P_{1}^{\prime}=0.445\right.$ day $)$ fits to the data are shown in the middle and bottom panels of Fig. 3, respectively. Both periodograms show residual peaks at about 5.2-5.4 $\mathrm{c} \mathrm{d}^{-1}$ with $1 \mathrm{c} \mathrm{d}^{-1}$ alias sidelobes. Non-linear least-square fitting of the residual $R V$ data led to a best-fit frequency of $f_{2}=5.273 \pm 0.03 \mathrm{c} \mathrm{d}^{-1}\left(P_{2}=0.190 \pm\right.$ 0.001 day) with a semi-amplitude of $3.9 \pm 0.59 \mathrm{~m} \mathrm{~s}^{-1}$. These peaks are also statistically significant. The false alarm probability (FAP) using the bootstrap technique yields FAP $=5 \times 10^{-6}$ (i.e. with $2 \times 10^{5}$ random data sets there was only one instance where the random periodogram had power exceeding that of the data). These residual $R V \mathrm{~s}$ folded with a $P_{2}=0.190$ day period are shown in Fig. 5.

The $R V$ data of Run 2 is much sparser and has variations that are marginally above that of the noise level. Even if a single stable period were present, there could be several periods that could fit these data equally well. We analyzed the data using both the Scargle periodogram and the Deeming DFT.

Figure 6 shows the Deeming (top panel) and the Scargle periodograms (bottom panel) of the $R V$ measurements from the second observing run. As expected, both show severe aliasing patterns. We note that the Lomb-Scargle periodogram shows the highest peak at $f_{3}^{\prime}=3.912 \mathrm{c} \mathrm{d}^{-1}\left(P_{3}^{\prime}=0.256\right.$ day $)$, while the Deeming periodogram gives highest peaks at $f_{3}=5.419 \mathrm{c} \mathrm{d}^{-1}$ $\left(P_{3}=0.185\right.$ day) or its $1 \mathrm{~cd}^{-1}$ sidelobe at $f_{3}^{\prime \prime}=4.42 \mathrm{~cd}^{-1}$ $\left(P_{3}^{\prime \prime}=0.226\right.$ days $)$. The FAP $\approx 5 \times 10^{-6}$ for the $f_{3}^{\prime}=3.912 \mathrm{c} \mathrm{d}^{-1}$ signal $\left(2 \times 10^{5}\right.$ random shuffles of a bootstrap). Both fits are shown in Fig. 2 and as phase-folded radial-velocity curves in Fig. 7. A 0.256 -day period provides a slightly better fit to the data with an rms scatter about the sine wave $\sigma=5.1 \mathrm{~m} \mathrm{~s}^{-1}$. The 0.185 -day period yields $\sigma=7.4 \mathrm{~m} \mathrm{~s}^{-1}$. However, both are well below our internal errors, which may be over estimated. 
Table 1. Relative $R V$ measurement for $\alpha$ Ari.

\begin{tabular}{|c|c|c|c|c|c|c|c|c|}
\hline $\begin{array}{c}\text { JD-2452000 } \\
\text { day }\end{array}$ & $\begin{array}{c}\Delta R V \\
\mathrm{~m} \mathrm{~s}^{-1}\end{array}$ & $\begin{array}{c} \pm \sigma \\
\mathrm{m} \mathrm{s}^{-1}\end{array}$ & $\begin{array}{c}\text { JD-2452000 } \\
\text { day }\end{array}$ & $\begin{array}{c}\Delta R V \\
\mathrm{~m} \mathrm{~s}^{-1}\end{array}$ & $\begin{array}{c} \pm \sigma \\
\mathrm{m} \mathrm{s}^{-1}\end{array}$ & $\begin{array}{c}\text { JD-2452000 } \\
\text { day }\end{array}$ & $\begin{array}{l}\Delta R V \\
\mathrm{~m} \mathrm{~s}^{-1}\end{array}$ & $\begin{array}{c} \pm \sigma \\
\mathrm{m} \mathrm{s}^{-1}\end{array}$ \\
\hline 948.10923 & 0.33 & 5.62 & 948.26981 & 13.92 & 6.66 & 950.16893 & -4.54 & 6.77 \\
\hline 948.11197 & 1.08 & 7.07 & 948.27123 & 19.64 & 6.23 & 950.20076 & -20.70 & 5.93 \\
\hline 948.11376 & 1.83 & 7.01 & 948.27266 & 18.93 & 5.13 & 950.20237 & -23.61 & 6.58 \\
\hline 948.11620 & 0.42 & 6.15 & 948.27266 & 16.71 & 5.42 & 950.20531 & -18.94 & 7.56 \\
\hline 948.11786 & 6.14 & 6.48 & 948.27408 & 15.73 & 5.77 & 950.20665 & -17.78 & 6.42 \\
\hline 948.11942 & -6.82 & 6.54 & 948.29048 & 10.51 & 5.75 & 950.20802 & -17.35 & 5.78 \\
\hline 948.12136 & -1.62 & 6.08 & 948.29221 & 14.14 & 6.49 & 950.20937 & -21.46 & 6.41 \\
\hline 948.12297 & 0.40 & 5.63 & 948.29377 & 11.96 & 7.68 & 950.21155 & -18.23 & 6.08 \\
\hline 948.12442 & 2.78 & 7.34 & 948.29513 & 15.17 & 9.08 & 950.21290 & -23.72 & 7.14 \\
\hline 948.12630 & -1.71 & 6.86 & 948.29650 & 10.97 & 7.50 & 950.21426 & -19.22 & 5.78 \\
\hline 948.12798 & 2.54 & 6.72 & 948.29786 & 8.58 & 7.61 & 950.21585 & -24.29 & 6.22 \\
\hline 948.12957 & 1.63 & 8.61 & 948.29958 & 11.42 & 5.74 & 950.21728 & -21.97 & 6.52 \\
\hline 948.13110 & 5.27 & 7.30 & 948.30098 & 12.82 & 9.52 & 950.21860 & -27.84 & 6.65 \\
\hline 948.13290 & 5.57 & 7.59 & 948.30256 & 12.97 & 7.30 & 950.21993 & -21.48 & 7.13 \\
\hline 948.13438 & 2.63 & 5.79 & 948.30453 & 8.59 & 7.65 & 950.22126 & -21.55 & 6.59 \\
\hline 948.13598 & 0.34 & 6.34 & 948.30604 & 6.00 & 7.10 & 950.24745 & -16.70 & 10.45 \\
\hline 948.13746 & 10.56 & 7.37 & 948.30746 & 11.69 & 7.58 & 950.24951 & -24.47 & 7.52 \\
\hline 948.13894 & 6.88 & 6.69 & 948.30889 & 15.47 & 6.84 & 950.25130 & -27.99 & 7.76 \\
\hline 948.14052 & 8.64 & 6.25 & 948.31072 & 7.43 & 7.22 & 950.25284 & -19.21 & 7.45 \\
\hline 948.17146 & 9.22 & 6.65 & 948.31220 & 16.10 & 6.37 & 950.25446 & -14.22 & 6.27 \\
\hline 948.17501 & 8.33 & 6.41 & 949.19486 & 0.52 & 9.47 & 950.25637 & -27.49 & 7.72 \\
\hline 948.17649 & 15.62 & 6.53 & 949.19746 & 2.04 & 10.03 & 950.29188 & -18.12 & 8.93 \\
\hline 948.17809 & 18.55 & 7.40 & 949.21917 & -1.10 & 7.38 & 950.29368 & -18.44 & 10.40 \\
\hline 948.17951 & 10.20 & 6.25 & 949.22054 & -0.77 & 6.97 & 950.29568 & -16.51 & 9.00 \\
\hline 948.18265 & 9.05 & 6.11 & 949.22190 & 3.00 & 7.20 & 950.29790 & -21.11 & 9.36 \\
\hline 948.18438 & 13.94 & 6.20 & 949.23297 & 3.32 & 7.51 & 950.30023 & -8.88 & 10.76 \\
\hline 948.18583 & 12.50 & 6.72 & 949.23465 & 2.66 & 7.97 & 950.30189 & -14.61 & 12.16 \\
\hline 948.18719 & 12.47 & 5.04 & 949.23608 & 4.23 & 7.16 & 975.99127 & 7.35 & 21.00 \\
\hline 948.18856 & 13.37 & 5.87 & 949.24710 & -0.89 & 7.52 & 976.00851 & 14.19 & 18.03 \\
\hline 948.18994 & 18.71 & 6.28 & 949.24857 & -2.59 & 7.84 & 976.02808 & 16.64 & 16.82 \\
\hline 948.19129 & 11.16 & 6.78 & 949.24999 & 5.33 & 8.04 & 976.04671 & 2.36 & 13.97 \\
\hline 948.19274 & 10.67 & 7.42 & 949.26332 & -5.24 & 7.61 & 976.06440 & -13.00 & 16.09 \\
\hline 948.19639 & 9.83 & 6.61 & 949.26493 & 5.25 & 8.10 & 976.09031 & -14.17 & 22.36 \\
\hline 948.19820 & 12.38 & 6.42 & 950.01919 & 7.28 & 6.97 & 976.09592 & -34.44 & 13.61 \\
\hline 948.20027 & 14.57 & 5.93 & 950.02127 & 11.06 & 6.49 & 976.09904 & -36.84 & 16.13 \\
\hline 948.20182 & 11.76 & 6.55 & 950.02267 & 6.94 & 6.24 & 977.99945 & -10.19 & 14.21 \\
\hline 948.20333 & 12.16 & 6.86 & 950.05321 & 0.95 & 6.11 & 978.00173 & -9.41 & 12.73 \\
\hline 948.20483 & 16.26 & 6.35 & 950.05496 & 5.26 & 6.98 & 978.00413 & -16.51 & 13.57 \\
\hline 948.20632 & 10.05 & 5.89 & 950.05634 & 4.35 & 6.56 & 978.00677 & -15.80 & 13.17 \\
\hline 948.25135 & 14.52 & 6.36 & 950.08323 & 7.72 & 6.46 & 978.12289 & -9.58 & 13.03 \\
\hline 948.25312 & 18.58 & 6.84 & 950.08487 & 2.05 & 6.96 & 978.12575 & -21.84 & 14.52 \\
\hline 948.25448 & 21.00 & 6.37 & 950.08625 & -1.59 & 6.46 & 978.12850 & -14.02 & 12.54 \\
\hline 948.25586 & 18.09 & 7.23 & 950.10334 & -4.77 & 7.60 & 978.13140 & -21.75 & 13.72 \\
\hline 948.25723 & 14.41 & 7.11 & 950.10493 & 11.44 & 6.63 & 978.13411 & -23.97 & 13.17 \\
\hline 948.25859 & 18.85 & 7.27 & 950.10630 & 4.44 & 7.68 & 981.05451 & -19.38 & 13.50 \\
\hline 948.26275 & 19.83 & 5.94 & 950.13615 & -6.50 & 7.58 & 981.05722 & -18.71 & 12.96 \\
\hline 948.26413 & 17.22 & 6.66 & 950.13786 & -0.41 & 7.55 & 981.06131 & -20.57 & 11.49 \\
\hline 948.26549 & 19.87 & 6.26 & 950.13959 & 4.21 & 7.46 & 981.06555 & -11.35 & 11.31 \\
\hline 948.26686 & 21.34 & 5.96 & 950.16535 & -5.03 & 7.28 & & & \\
\hline 948.26822 & 19.01 & 5.80 & 950.16744 & -12.82 & 7.18 & & & \\
\hline
\end{tabular}

The Run 2 data are too sparse to determine which period is correct, and the amplitude of any $R V$ variations is too low for us to be completely confident of the $P_{3}$ period. It is evident, however, that $P_{1}$ was not present during the second run. Figure 8 shows the $R V$ s from Run 2 phased to the 0.57 day period and the 0.82 day period. There is no evidence of sinusoidal variations. If $P_{1}$ were present, then it had a significantly lower amplitude than the previous observing run. We tend to consider $P_{3}=0.185 \mathrm{~d}$ to be real as it is consistent with the second period $P_{2}=0.190$ day that was found in the first run.

The absence of the 0.571 ( 0.445 or 0.821$)$ day pulsations in Run 2 and the coincidence of the short periodic $(0.185 \mathrm{~d})$ pulsations in both runs may be indications of multi-periodic oscillations that either switch modes or have temporal variations in the pulsation amplitudes.

\section{Discussion}

The short period variability of $\alpha$ Ari can only result from stellar oscillations. In order to determine the pulsation mode, we use the classical determination of the pulsation constant

$Q=P\left(M / M_{\odot}\right)^{0.5}\left(R / R_{\odot}\right)^{-1.5}$

where $M, R$ and $M_{\odot}, R_{\odot}$ are the masses and radii of the star and Sun, respectively. 


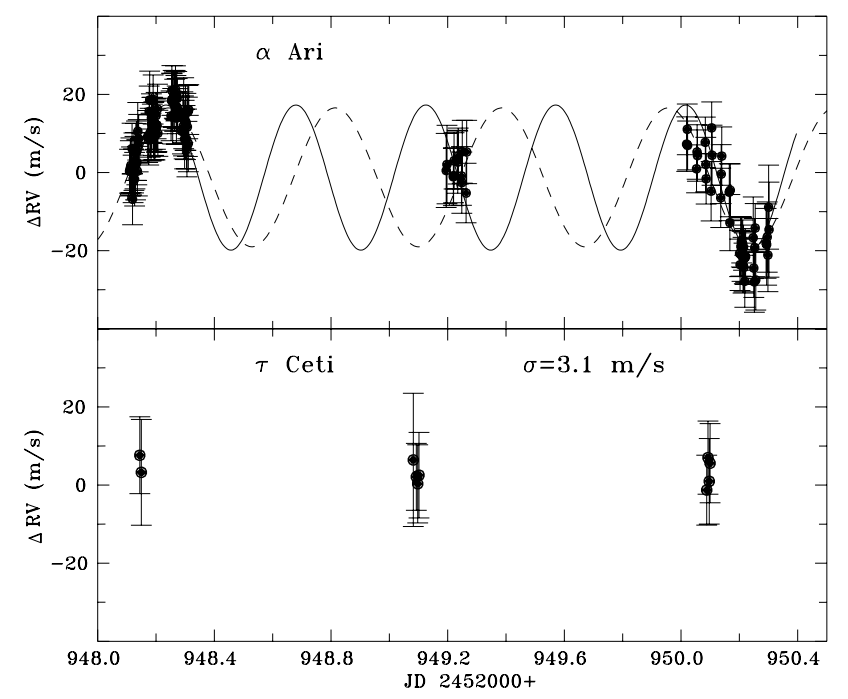

Fig. 1. JD $=2452948-2452950$ (Run 1) relative $R V$ measurements of $\alpha$ Ari (top panel) and the radial velocity standard star $\tau$ Cet (bottom panel). The solid line shows a sine wave with a period of 0.445 days and the dashed line a sine fit a period of 0.571 days.

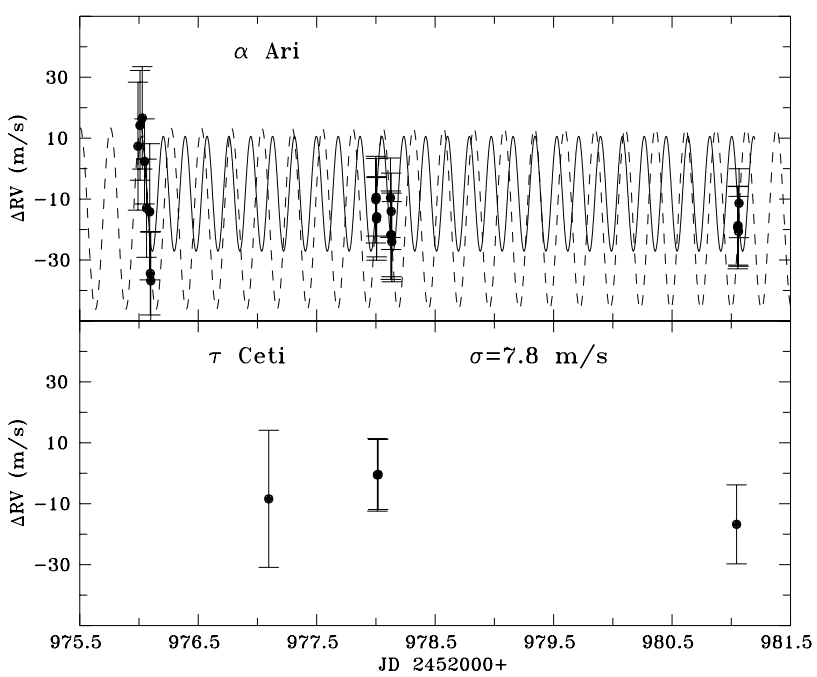

Fig. 2. JD $=2452975-2452981$ (Run 2) relative $R V$ measurements of $\alpha$ Ari (top panel) and the radial velocity standard star $\tau$ Cet (bottom panel). The solid line shows the $P=0.184$-day fit and the dashed line is a sine-fit with $P=0.256$ days.

This star has been the subject of several spectroscopic investigations. Gray et al. (2002) determined $T_{\mathrm{e}}=4481 \pm 11 \mathrm{~K}$ and $[\mathrm{Fe} / \mathrm{H}]=-0.221 \pm 0.036$. These values are in agreement with $T_{\mathrm{e}}=4480 \mathrm{~K}$ and $[\mathrm{Fe} / \mathrm{H}]=-0.25 \pm 0.09$ as found by McWilliam (1990). These effective temperature determinations are in excellent agreement with $T_{\mathrm{e}}=4490 \pm 61 \mathrm{~K}$ determined using the Infra Red Flux Method (Alonso et al. 1999).

Mozurkewich et al. (1991) measured an angular diameter for $\alpha$ Ari of $6.412 \pm 0.064$ mas at $800 \mathrm{~nm}$. The Hipparcos parallax of $49.48 \pm 0.99$ mas results in a stellar radius of $R=13.9 \pm 0.3 R_{\odot}$. Using $\log g=2.57 \pm 0.2$ (McWilliam 1990) results in a stellar mass as $M=2.60 \pm 0.2 M_{\odot}$. For these mass and radius the pulsation constant $(Q)$ for a period $P_{1}=0.571$ (alternatively $P_{1}^{\prime}=0.445$ or $\left.P_{1}^{\prime \prime}=0.821\right)$ days is equal to $Q_{1}=0.0177$ day $\left(Q_{1}^{\prime}=0.0138\right.$ or $Q_{1}^{\prime \prime}=0.0255$, respectively $)$, while pulsation constant for the secondary period $P_{2}=0.190$ is equal to $Q_{2}=0.0059$ days.

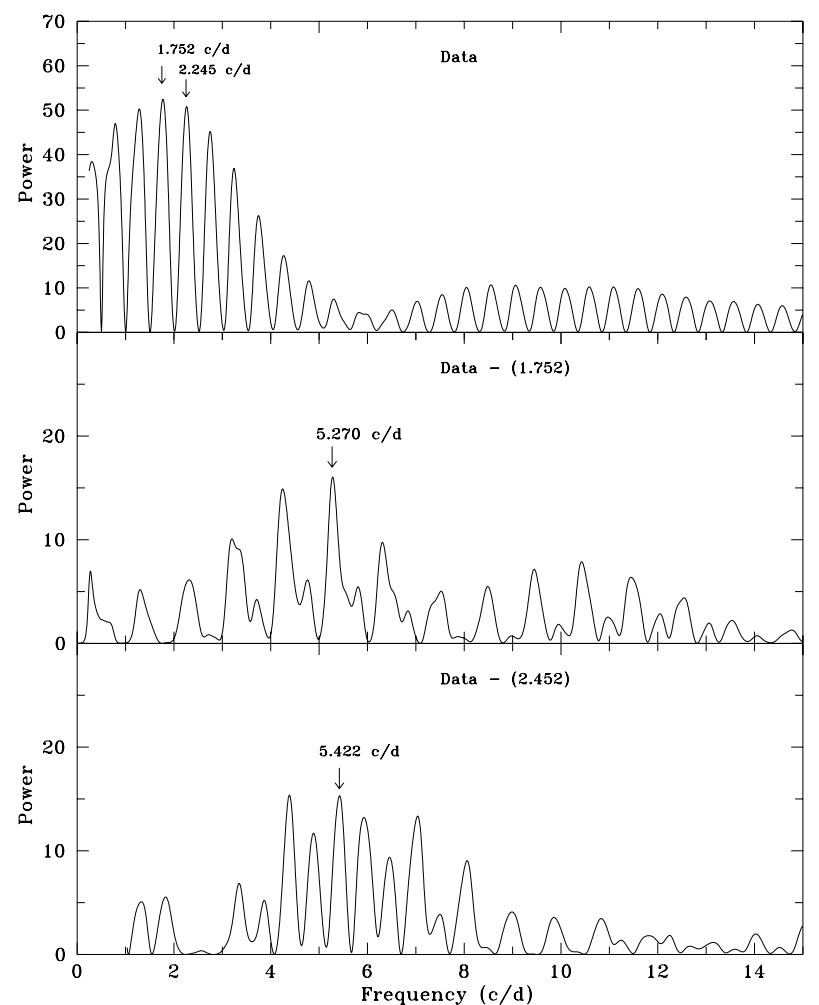

Fig. 3. Periodograms of the JD $=2452948-J D 2452950 R V$ data of $\alpha$ Ari. Top panel: the periodogram of the original data. The arrows show the positions of the two highest frequencies $f_{1}=1.752 \mathrm{~cd}^{-1}$ and $f_{1}=2.245 \mathrm{c} \mathrm{d}^{-1}$. Middle panel: the periodogram of residuals after removing the $f_{1}=1.752 \mathrm{~cd}^{-1}$ signal. Bottom panel: the periodogram of the residual $R V \mathrm{~s}$ after removing the contribution of the $f_{1}=2.245 \mathrm{c} \mathrm{d}^{-1}$ signal. The periodograms of the residual velocities after subtracting the dominant period show significant power in the frequency range $5.2-5.4 \mathrm{c} \mathrm{d}^{-1}$.

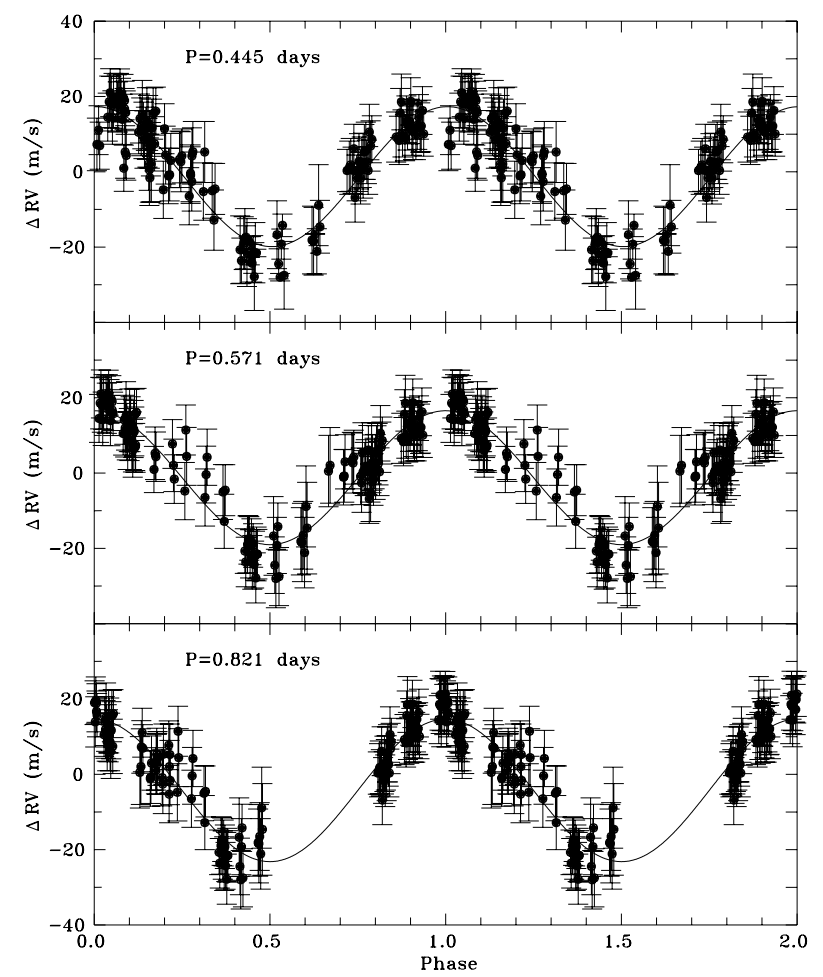

Fig. 4. The $R V$ curve folded with $P_{1}=0.445,0.571$, and 0.821 day periods (top to bottom). 
Table 2. The pulsation constants $Q$ (days) for the fundamental and $n=1-12$ overtone pulsations of $4.0 M_{\odot}, \log L / L_{\odot}, \log T_{\text {eff }}=3.6633$, $\log g=2.139$ K-giant model of Guenther et al. (2000).

\begin{tabular}{ccccccccccccccc}
\hline \hline$n$ & $\mathrm{~F}$ & 1 & 2 & 3 & 4 & 5 & 6 & 7 & 8 & 9 & 10 & 11 & 12 \\
$Q$ & 0.0578 & 0.0309 & 0.0221 & 0.0173 & 0.0145 & 0.0125 & 0.0108 & 0.096 & 0.0086 & 0.0078 & 0.0071 & 0.0066 & 0.0061 \\
\hline
\end{tabular}

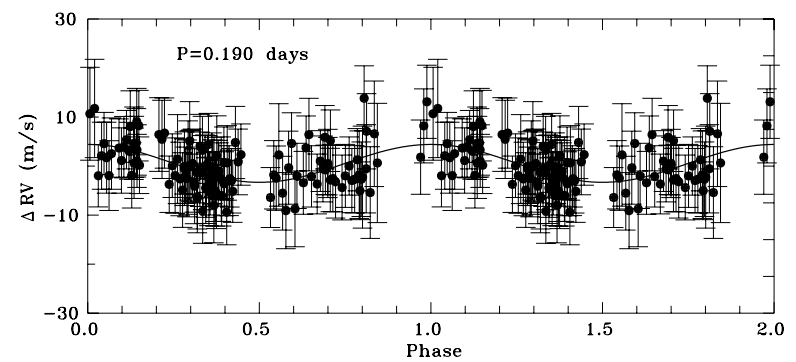

Fig. 5. Phase diagram of the $R V$ residuals from Run 1 after removal of the $P_{1}=0.571$ component. These are phased to a period of $P_{1}=$ 0.190 days.

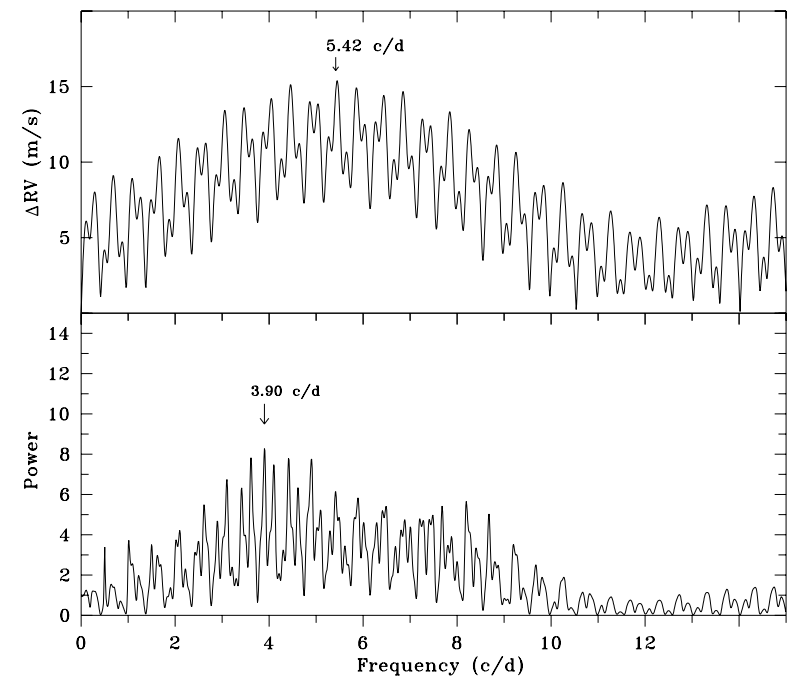

Fig. 6. DFT amplitude spectra (top panel) and Scargle periodogram (bottom panel) of the JD $=2452975-\mathrm{JD} 2452981 R V$ data of $\alpha$ Ari. The arrows show the position of the highest peaks.

For mode identification we used the predicted oscillation frequencies for radial modes from the theoretical models of Guenther et al. (2000), which are appropriate for a K giant star with a mass of 4.0-5.0 $M_{\odot}$. This comparison may be appropriate for $\alpha$ Ari with its lower mass due to the weak dependence of $Q$ with stellar mass. Table 2 gives the calculated pulsational constants for the $4.0 M_{\odot}$ model ranging from the fundamental mode to $n=12$ radial overtones.

Comparisons of theoretical constants with observed ones suggests that the $P_{1}=0.571\left(P_{1}^{\prime}=0.445\right.$ or $\left.P_{1}^{\prime \prime}=0.821\right)$ day period corresponds approximately to the $n \simeq 3$ radial overtone pulsations, while the approximate overtone identification for secondary period $P_{2}=0.190$ can be related to the $n \geq 12$ overtone. Up to now the high-overtone pulsations have not been observed in K-giants.

The short-period oscillations detected in $\alpha$ Ari are also comparable to those found in $\beta$ Oph $(P=0.256$ days, Hatzes \& Cochran 1994b), as well as with the short-period $0.541 \mathrm{~d}\left(1.846 \mathrm{~cd}^{-1}\right), 0.248 \mathrm{~d}\left(4.028 \mathrm{~cd}^{-1}\right)$, and $0.162 \mathrm{~d}$ $\left(6.167 \mathrm{~cd}^{-1}\right)$ variability found in K-giant GSC 09137-03505

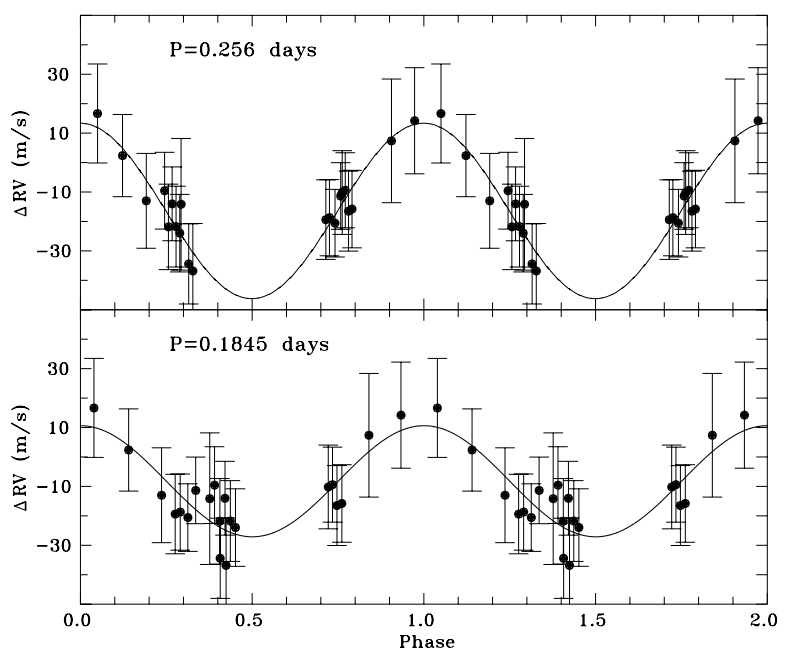

Fig. 7. Phase-folded with $P=0.256$ day (top panel) and with $P=$ 0.184 day (bottom panel) $R V$ curve for the observations taken over JD = 2452 975-JD 2452981.

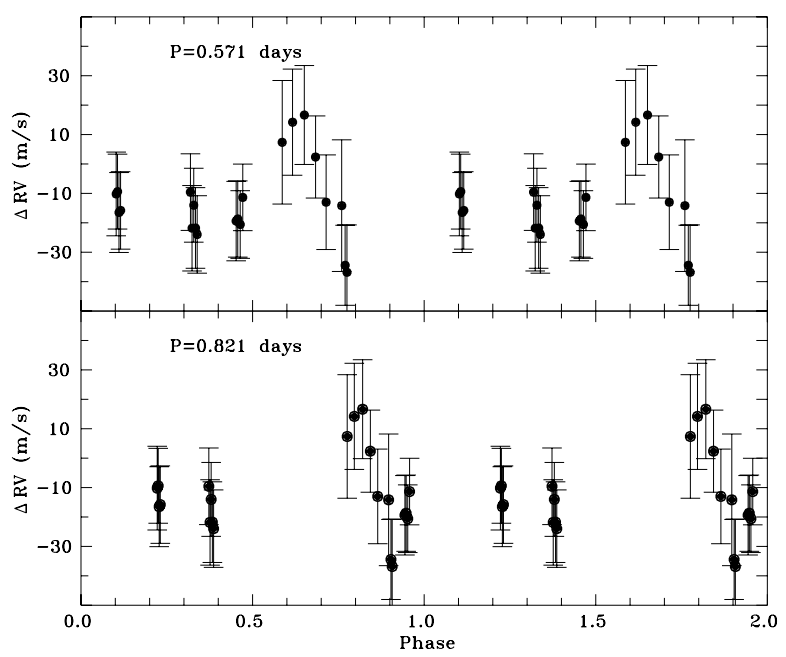

Fig. 8. $R V$ data from JD $=2452975-J D 2452981$ phase-folded with a $P_{1}=0.571$ day (top panel) and $P_{1}=0.821$ day (bottom panel).

(Kallinger et al. 2005). All three frequencies in K-giant GSC 09137-03505 were found, however, to lie above the acoustic cutoff frequency for evolutionary models fitting this star.

Marginal evidence of additional high-frequency (up to $6.8 \mathrm{c} \mathrm{d}^{-1}$ ) oscillations in Arcturus was earlier reported by Belmonte et al. (1990). This work was based on the analysis of $R V$ data over two weeks that had a mode detection threshold $2 \mathrm{~m} \mathrm{~s}^{-1}$. They interpreted variability as due to high-overtone $n \approx 16$ modes. However, space-born photometric observations of Arcturus (Retter et al. 2003) show the high-frequency cut-off of $0.9 \mathrm{~cd}^{-1}$ and do not confirm the Belmonte et al. (1990) high-frequency results.

From a theoretical point of view, the stability analysis of the $2.0 \quad M_{\odot}$ and $4 \quad M_{\odot}$ models of K-giants by 
Dziembowski et al. (2001) predicted both the instability of $n=$ $1-18$ overtones of low degree $l=0-2$ modes and the preferable excitation and higher amplitudes of $n \geq 5$ overtones compared to the fundamental mode. In this sense, the detection of 0.571 day, low-overtone oscillations and a 0.190-day, $n \simeq 12$ oscillations in $\alpha$ Ari agrees with the theoretical work of Dziembowski et al. (2001) and provides additional observational evidence of the co-excitation of low and high-overtone oscillations in $\mathrm{K}$ giants. More intense $R V$ observations of $\mathrm{K}$-giants with high time resolution $\left(10^{-2}\right.$ day) are needed to understand the full range of the mode frequencies a K-giant may exhibit. The majority of previous $R V$ studies of $\mathrm{K}$-giants have had data sampling that could only detect periods of a few to several hundred days. Observations with better time sampling may reveal that other K-giants show relatively short periods or that previous longer periods were actually aliases of much shorter ones.

\section{Conclusion}

The radial velocities obtained with new echelle-spectrometer BOES of the 1.8-m telescope at Bohyunsan Optical Astronomy Observatory demonstrate the capability of relative radial velocity measurements to a precision of $3 \mathrm{~m} \mathrm{~s}^{-1}$.

As a first result of a survey of K-giants, we detected multiperiodic oscillations in $\alpha$ Ari with periods $P_{1}=0.571$ day (or a 0.871 and 0.445 day aliases) and $P_{2}=0.190$ day. Because of the short time scales, this variability can only be the result of stellar oscillations. We find that the dominant oscillation period of 0.571 days is consistent with the $n \simeq 3$ overtone mode, while the second period may be due to high $(n \geq 12)$ overtone pulsations. Similar to the earlier known K-giant pulsators, $\alpha$ Ari shows amplitude variability and switching of pulsation modes. Our observations add to the growing evidence that pulsations are common among $\mathrm{K}$ giant stars and that these are stochastically excited.
Acknowledgements. K.M.K. acknowledges the financial support by the KASI (Korea Astronomy and Space Science Institute) grant 2006-01-310-01 for this work. D.E.M. acknowledges his work as part of the research activity of the Astrophysical Research Center for the Structure and Evolution of the Cosmos (ARCSEC), which is supported by the Korean Science and Engineering Foundation. A.P.H. acknowledges the support of grant 50OW0204 from the Deutsches Zentrum für Luft- und Raumfahrt e.V. (DLR).

\section{References}

Alonso, A., Arribas, S., \& Martinez-Roger, C. 1999, A\&A, 139, 335

Belmonte, J. A., Jones, A. R., Palle, P. L., \& Roca Cortes, T. 1990, ApJ, 358, 595 Butler, R. P., Marcy, G. W., Williams, E., et al. 1996, PASP, 108, 500

Buzasi, D., Catanzarite, J., Laher, R., et al. 2000, ApJ, 532, L133

Deeming, I. J. 1975, Ap\&SS, 36, 136

Dziembowski, W. A., Gough, D. O., Houdek, G., \& Sienkiewicz, R. 2001, MNRAS, 328, 601

Guenther, D. B., Demarque, P., Buzasi, D., et al. 2000, ApJ, 530, L45

Gray, D. F., Scott, H. R., \& Postma, J. E. 2002, PASP, 114, 536

Hatzes, A. P., \& Cochran, W. D. 1993, ApJ, 413, 339

Hatzes, A. P., \& Cochran, W. D. 1994a, ApJ, 422, 369

Hatzes, A. P., \& Cochran, W. D. 1994b, ApJ, 432, 763

Hatzes, A. P., \& Cochran, W. D. 1998, Cool Stars Stellar Systems and the Sun, ed. R. A. Donahue, \& J. A. Bookbinder, ASP Conf. Ser., 154, 311

Kallinger, Th., Zwintz, K., Pamyatnykh, A. A., Guenther, D. B., \& Weiss, W. W. 2005, A\&A, 433, 267

Kim, K. M., Jang, B. H., Han, I., et al. 2002, J. Kor. Astron. Soc., 35, 221

Kürster, M., Schmitt, J. H. M. M., Cutispoto, G., \& Dennerl, K. 1997, A\&A, 320,831

Lomb, N. R. 1976, Ap\&SS, 39, 477

McWilliam, A. 1990, ApJS, 74, 1075

Merline, W. J. 1995, Ph.D. Thesis, The University of Arizona, Tucson, AZ

Mozurkewich, D., Johnston, K. J., Simon, R. S., et al. 1991, AJ, 101, 2207

Murdoch, K. A., Hearnshaw, J. B., \& Clark, M. 1993, ApJ, 413, 349

Retter, A., Bedding, T. R., Buzasi, D., \& Kjeldsen 2003, ApJ, 591, L151

Scargle, J. D. 1982, ApJ, 263, 835

Smith, P. H., McMillan, R. S., \& Merline, W. J. 1987, ApJ, 317, L79

Valenti, J. A., Butler, R. P., \& Marcy, G. W. 1995, PASP, 107, 966

Walker, G. A. H., Yang, S., Campbell, B., \& Irwin, A. W. 1989, ApJ, 343, L21 